\title{
Real-Time Thevenin Impedance Computation
}

\section{Sommer, Stefan Horst; Jóhannsson, Hjörtur}

Published in:

2013 IEEE PES Innovative Smart Grid Technologies

Link to article, DOI:

10.1109/ISGT.2013.6497824

Publication date:

2013

Link back to DTU Orbit

Citation (APA):

Sommer, S. H., \& Jóhannsson, H. (2013). Real-Time Thevenin Impedance Computation. In 2013 IEEE PES Innovative Smart Grid Technologies (pp. 6497824). IEEE. https://doi.org/10.1109/ISGT.2013.6497824

\section{General rights}

Copyright and moral rights for the publications made accessible in the public portal are retained by the authors and/or other copyright owners and it is a condition of accessing publications that users recognise and abide by the legal requirements associated with these rights.

- Users may download and print one copy of any publication from the public portal for the purpose of private study or research.

- You may not further distribute the material or use it for any profit-making activity or commercial gain

- You may freely distribute the URL identifying the publication in the public portal

If you believe that this document breaches copyright please contact us providing details, and we will remove access to the work immediately and investigate your claim. 


\title{
Real-Time Thevenin Impedance Computation
}

\author{
Stefan Sommer \\ Hjörtur Jóhannsson \\ Department of Electrical Engineering, Technical University of Denmark \\ Email: shso@elektro.dtu.dk / hj@elektro.dtu.dk
}

\begin{abstract}
Stable and secure operation of power systems becomes increasingly difficult when a large share of the power production is based on distributed and non-controllable renewable energy sources. Real-time stability assessment is dependent on very fast computation of different properties of the grid operating state, and strict time constraints are difficult to adhere to as the complexity of the grid increases. Several suggested approaches for real-time stability assessment require Thevenin impedances to be determined for the observed system conditions. By combining matrix factorization, graph reduction, and parallelization, we develop an algorithm for computing Thevenin impedances an order of magnitude faster than previous approaches. We test the factor-and-solve algorithm with data from several power grids of varying complexity, and we show how the algorithm allows realtime stability assessment of complex power grids at millisecond time scale.
\end{abstract}

\section{INTRODUCTION}

Efforts on de-carbonizing the power system often imply a shift from centralized and controllable energy production to distributed and non-controllable renewable energy sources. This shift makes stable and secure operation of power systems an increasingly challenging task.

In traditional power systems, stability could be assessed offline and sensitivities to various contingencies established by running time-consuming simulations. Multiple factors of the future power system challenge this approach: the complexity of the power grid will rise with increased de-centralization resulting in increased computational burden and longer runtime for simulations; and the power system should be able to operate under rapidly changing conditions for example due to inclusion of weather dependent energy sources. Large fluctuations of the system operating point can be common, and in combination these factors will likely make the results of conventional off-line stability assessment obsolete even before the time-domain simulation has completed. The need for realtime transient stability assessment has therefore been noted by several sources [1].

In this paper, we develop a fast algorithm for real-time computation of Thevenin impedances in complex power systems. Because several approaches for real-time stability assessment require knowledge of Thevenin impedances, the algorithm makes the use of these approaches feasible for complex power systems at increased time resolution. The factor-and-solve

This work is part of the Secure Operation of Sustainable Power Systems (SOSPO) project with support from the Danish Strategic Research Council (DSF). algorithm can therefore play a key role in concrete implementations of these transient stability assessment methods. We give theoretical arguments for why poor performance is observed for previous approaches, we exploit parallelism in parts of the algorithm to obtain a good balance between serial and parallel computation, and we evaluate the developed method on several complex power systems. Thus, we show how a key property of the power system state can be computed at millisecond scale and used for real-time stability assessment.

\section{A. Paper Overview}

The paper starts with a short description of approaches to real-time transient stability assessment. In Section III, we describe the problem of computing Thevenin impedances and previous approaches. The LU-factorization of the network admittance matrix takes a key role, and we progress to discussing it and node elimination strategies in the following section. We develop the factor-and-solve algorithm in Section VI and evaluate its performance and characteristics in the last section. The paper ends with concluding remarks.

\section{BACKGROUND}

Transient stability assessment is challenged both by a shift in time-scale and by increase in the complexity of power systems. With conventional power systems and centralized production, a relatively stable and controllable operating point allowed off-line stability evaluations to remain valid for hours. With de-centralization, weather dependence, and reduced control, stability of the rapidly fluctuating operating point must be evaluated at a much shorter time-scale, preferably in real-time.

At the same time, the complexity of power systems increases. Renewable energy sources add to the number of buses in the system, and power systems becomes increasingly interconnected to even out differences in the production of e.g. weather dependent sources. It is no longer sufficient to consider networks regionally due to increasing number of interconnections across national borders. This in turn requires real-time computation on networks with thousands of buses and tens of thousands of branches.

Real-time monitoring and control is in particular enabled by the advent of phasor measurement units (PMUs, [2], [3]). A few methods have been developed for real-time stability assessment using PMU data. In [4], an existing method is adapted for real-time use while [5], [6] propose a entirely new approach exploiting analytically derived expressions for stability boundaries [7]. See also [8] for a combined offline/on-line approach. The adaptability of existing off-line 
approaches to real-time computation and the dependence on grid complexity is surveyed in [9].

Thevenin impedance calculations constitutes a major component of the stability assessment in [6]. Several additional methods base voltage stability monitoring on local measurements and Thevenin impedances [10], [11], [12], [13].

Computations involving power grids are often performed on matrices representing the connections and state of the of the network. Typical computations involves factorizing these matrices using solvers that are particularly suited for sparse network computations [14]; relaxation methods [15]; and graph reduction algorithms [16]. See also [17] for comparison of factorization and relaxation methods.

A full time-domain simulation will typically simulate a differential-algebraic system. Thevenin impedance computations differ by only considering the algebraic part of network equations and the computational effort is therefore significantly reduced. This makes Thevenin impedance computations applicable as parts in real-time stability assessment methods. The computational aspects of dealing with this particular system of equations are different than when using the full differential-algebraic system, and the algorithm developed here reflects the real-time requirement.

\section{THEVENIN IMPEDANCES}

Consider a power grid consisting of $N$ nodes with the voltage at $M \leq N$ nodes being kept constant by means of voltage control equipment. Letting $Y$ denote the system admittance matrix, the system node voltage equation is

$$
I=Y V \text {. }
$$

The $M$ voltage controlled nodes ( $v c \mathrm{~s})$ and the $N-M$ nodes of non-controlled voltage (ncs) can be ordered so that the $n c$ s and $v c$ s are numbered by indices $1, \ldots, N-M$ and $N-$ $M+1, \ldots, N$, respectively. The system admittance matrix then takes the form

$$
Y=\left(\begin{array}{cc}
Y_{n c} & Y_{\text {link }} \\
Y_{\text {link }}^{T} & Y_{v c}
\end{array}\right)
$$

where $Y_{n c}$ denotes the admittance matrix of only the noncontrolled $n c$ part of the system, $Y_{v c}$ denotes the admittance matrix of the voltage controlled $v c$ part, and $Y_{\text {link }}$ encodes the links between the $n c$ and $v c$ parts of the network.

For each node $k$ of the $v c$ s, we are interested in computing the Thevenin impedance for the node, i.e. the impedance seen from node $k$ when all $v c$ nodes besides node $k$ node are shorted. This situation can be modeled by removing all $v c$ nodes besides $k$ from the system, and the Thevenin impedance $Z_{\text {th }, k}$ can then be obtained by inverting the resulting admittance matrix. We let $Y_{\text {link }, \cdot k}$ denote the column of the link matrix $Y_{\text {link }}$ corresponding to the $k$ th node, and, correspondingly, we let $Y_{\text {link,k}}^{T}$. denote the row of the transpose link matrix corresponding to the $k$ th node. Letting $Y_{(k, k)}$ denote the $k$ th diagonal element of $Y$, we define

$$
Y_{k}=\left(\begin{array}{cc}
Y_{n c} & Y_{\text {link }, \cdot k} \\
Y_{\text {link }, k}^{T} & Y_{(k, k)}
\end{array}\right),
$$

i.e. the admittance matrix with all $v c$ nodes but node $k$ removed. The Thevenin impedance $Z_{t h, k}$ then equals the last diagonal element of the inverted matrix $Y_{k}^{-1}$.

A naive algorithm for computing $Z_{t h, k}$ for all $v c$ nodes would set up and invert $Y_{k}$ for each $k=N-M+1, \ldots, N$. This is a very inefficient approach and in practice infeasible for real-time computation since the number of arithmetic operations required for inverting a matrix has complexity $O\left(n^{3}\right)[18]^{2}$. The fact that the upper left $(N-M) \times(N-M)$ submatrix of $Y_{k}$ does not depend on $k$ strongly suggests that we can be more efficient.

\section{IMPEDANCES FROM LU-FACTORIZATIONS}

The LU-factorization [18], [19] splits a matrix into a product of a lower diagonal and an upper diagonal matrix, e.g. for the admittance matrices $Y$ and $Y_{k}$, factorizations

$$
Y=L U \text { and } Y_{k}=L_{k} U_{k}
$$

can be obtained. In particular, the inverse of $Y_{k}$ is given by $Y_{k}^{-1}=U_{k}^{-1} L_{k}^{-1}$. It is conventional to let the diagonal elements of $L_{k}$ be all 1 which then implies that the Thevenin impedance $Z_{t h, k}$ is given by inverse of the last diagonal element of $U_{k}$, i.e. $Z_{t h, k}=\left(U_{k,(N-M+1, N-M+1)}\right)^{-1}$.

It is shown in [5] that this diagonal element and hence $Z_{t h, k}$ can be recovered from the factorization $L, U$ of the full admittance matrix $Y$ by the formula

$$
U_{k,(N-M+1, N-M+1)}=Y_{(k, k)}-\hat{L}_{k} \cdot \hat{U}_{\cdot k}
$$

with the last term being the inner product between the entries $1, \ldots, N-M$ of the $k$ th row of $L$ and of the $k$ th column of $U$. The advantage of using this relation is that only one matrix, $Y$, needs to be factorized in order to compute $Z_{t h, k}$ for all $v c$ nodes, i.e. for all $k=N-M+1, \ldots, N$. Although $Y$ is larger than $Y_{k}$, this offers a substantial reduction in computational effort. In [5], $L U$-factorization of $Y$ and (3) is used for computing Thevenin impedances. We analyze this method below in order to develop a more efficient approach, and we use the method as basis for the comparisons in the experiment section.

\section{A. Sparsity, Ordering and Fill-Ins}

Due to the very high sparsity of network matrices, $L U$ factorization is in general a very efficient procedure. Though the worst case performance is $O\left(N^{3}\right)$, the complexity is in practice close to linear $[17]^{3}$. This complexity can be reached with appropriate ordering of the matrix rows and columns and with specialized solvers. In line with [17], we use the KLU solver [14] that is particularly optimized for matrices with sparsity structure equivalent to power network matrices.

A key factor in achieving close to linear complexity in the factorization is minimizing the number of fill-ins, non-zero elements of the factors $L$ and $U$ that are not present in $Y$. The number of fill-ins is very dependent on the ordering of

\footnotetext{
${ }^{2}$ Instead of inverting $Y_{k}$, a linear system could be solved to get the result. This, however, does not change the $O\left(n^{3}\right)$ complexity.

${ }^{3}[17]$ experimentally assesses the complexity to $O\left(N^{\alpha}\right), \alpha \approx 1.2$.
} 

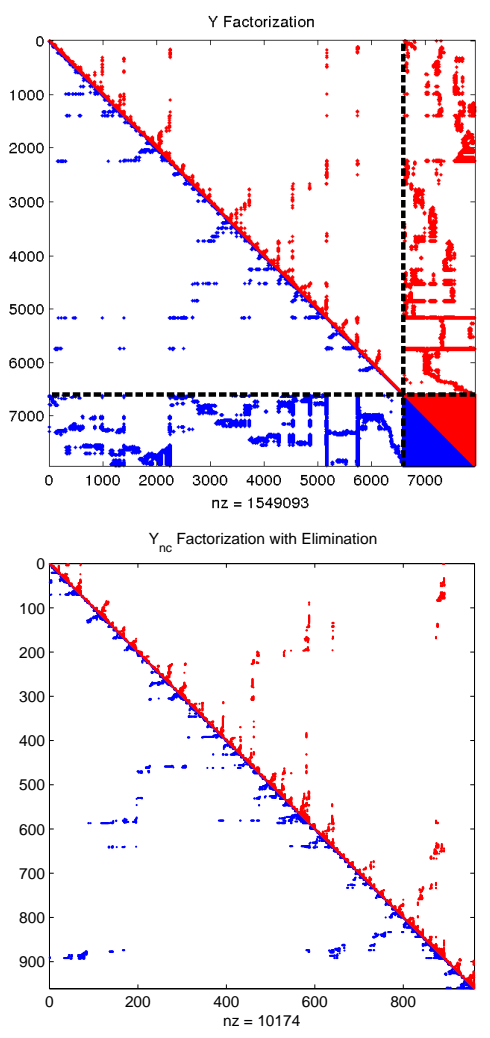

Figure 1. Sparsity patterns of (top) the LU-factorization of the full admittance matrix $Y$ with ordering placing the voltage controlled nodes ( $v c s$ ) to the right; and (bottom) the LU-factorization of the $Y_{n c}$ submatrix after application of node elimination. The excessive fill-ins in the lower right $v c$-part of the full factorization (top) slows down the algorithm. In contrast, the factorization of the reduced matrix (bottom) can be done with very limited fill-in and consequently very fast factorization. The factor-and-solve algorithm reduces both the dimension of the matrix to be factored (in this case from $7917 \times 7917$ to $960 \times 960$ ) and the number of non-zeros in the factors (from 1549093 to 10174) thus providing a great reduction in computational time.

the matrix $Y$. For network matrices, ordering algorithms like Approximated Minimum Degree (AMD, [20]) and variants ensure a very low degree of fill-in. The number of both nonzeros in $Y$ and additional fill-ins are in practice close to linearly correlated with $N$ which implies the close to linear complexity of the factorization.

In (2), we used an ordering with the ncs occurring with lower indices than the $v c$ s. This ordering is required for the relation (3) that allows us to extract the Thevenin impedances. To adhere to this indexing convention, [5] applies AMD ordering to the submatrices $Y_{n c}$ and $Y_{v c}$ individually before combining them to obtain the full matrix $Y$ as in (2). The result of this partial ordering strategy is that the upper left part of the factors $L, U$ becomes adequately sparse but the lower right part of the factors unfortunately contains a very large number of fill-ins. This problem that slows down the algorithm considerably is illustrated in Figure 1. In the next section, we give a theoretical explanation for the occurrence of the fill-ins, and we show how an improved Thevenin impedance algorithm avoids this problem.

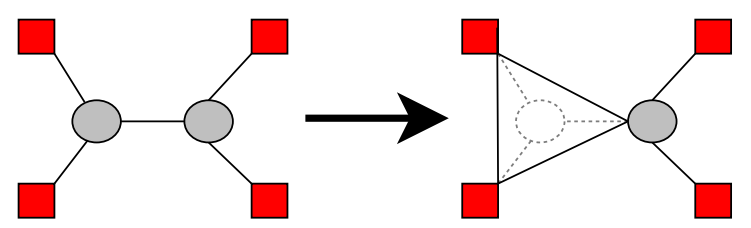

Figure 2. Elimination of one of two interior nodes in a six node network. The node to be eliminated has degree three and with three new branches added to the reduced network, the total number of branches is kept constant. This preserves the sparsity. Elimination of nodes with higher degree will result in an increased number of branches reducing sparsity.

\section{Schur Complement AND Node Elimination}

With system loads represented by their admittance values, no current enters the nodes of non-controlled voltage (ncs), and the network equation (1) can be stated as

$$
\left(\begin{array}{c}
0 \\
I_{v c}
\end{array}\right)=\left(\begin{array}{cc}
Y_{n c} & Y_{\text {link }} \\
Y_{\text {link }}^{T} & Y_{v c}
\end{array}\right)\left(\begin{array}{l}
V_{n c} \\
V_{v c}
\end{array}\right) .
$$

Using the Schur complement [21], [16]

$$
S=Y_{v c}-Y_{\text {link }}^{T} Y_{n c}^{-1} Y_{\text {link }}
$$

of $Y_{v c}$, the $v c$-part of the solution to (4) can be obtained by solving the reduced system $I_{v c}=S V_{v c}$. The Schur complement can in addition be obtained by successively eliminating nodes from the system and creating reduced admittance matrices. For each node to be eliminated as illustrated in Figure 2, the new admittance matrix is given by the formula

$$
Y_{(i, j)}^{\text {new }}=Y_{(i, j)}-\frac{Y_{(i, N)} Y_{(N, j)}}{Y_{(N, N)}},
$$

and $S$ is the matrix resulting from eliminating all ncs. Confer [16] for more information on node elimination and network reduction.

\section{A. Why Y Should Not Be Factored}

When eliminating nodes, branches are added to the resulting network, and in the completely reduced network consisting of all $v c$ s, all pairs of nodes are in general connected by branches. The Schur complement $S$ is therefore a dense matrix.

In [22], [23], it is observed that if a matrix with the block structure in (2) is $L U$-factored, the product of the lower right blocks $L_{v c}, U_{v c}$ of the factors $L, U$ corresponding to the $v c$ s provide the Schur complement of $Y_{v c}$ directly, i.e. $S=L_{v c} U_{v c}$. This provides a way to compute and factor $S$ but it also tells us why the large number of fill-ins are observed when computing Thevenin impedances with the method of [5] that uses factorization of the full matrix $Y$ : because $S$ is dense, the factors $L_{v c}$ and $U_{v c}$ will in general not be sparse ${ }^{4}$, and $L_{v c}, U_{v c}$ are precisely the lower right blocks of the factors $L, U$ where the excessive number of fill-ins occur. Indeed, any fixed bound on the maximum node degree in both $L_{v c}$ and $U_{v c}$ would imply that the number of non-zeros in $S$ would grow linearly with

\footnotetext{
${ }^{4}$ The product of sparse matrices can be dense. However, if we for example limit the node degree of the networks represented by the factors, the product will be sparse.
} 
the number of $v c$ s, i.e. $M$. Since $S$ is dense, the number of non-zeros grow quadratically, $\operatorname{nnz}(S) \approx M^{2}$, implying that no such bound can exist.

\section{FACTOR-AND-SOLVE THEVENIN IMPEDANCE ALGORITHM}

We here derive a fast algorithm for computing Thevenin impedances without factoring the full admittance matrix $Y$ and thereby avoiding the excessive fill-in in the Schur complement part of the factors. We denote the resulting algorithm factorand-solve relating to its composition of two individual steps.

We derive the algorithm by coupling a variant of the relation (3) with the structure of left-looking LU-factorization algorithms. First, a close variant of (3) for computing $U_{k,(N-M+1, N-M+1)}$ uses $Y_{k}$ instead of $Y$. Using the factorization $Y_{k}=L_{k} U_{k}$, we have

$$
U_{k,(N-M+1, N-M+1)}=Y_{(k, k)}-\hat{L}_{k,(N-M+1)} \cdot \hat{U}_{k, \cdot(N-M+1)}
$$

where the notation in the rightmost term denotes the inner product between entries $1, \ldots, N-M$ of the last row of $L_{k}$ and of the rightmost column of $U_{k}$. The advantage of using this formula is that $\hat{L}_{k,(N-M+1)}$. and $\hat{U}_{k, \cdot(N-M+1)}$ can be obtained from a factorization $Y_{n c}=L_{n c} U_{n c}$ of the $n c$-part of $Y$ only.

We now consider iterations of left-looking LU-factorization algorithms [19]. With this class of algorithms, the $N-M+1$ columns in a factorization $Y_{k}=L_{k} U_{k}$ are computed iteratively from left to right, i.e. starting with column 1 and ending with column $N-M+1$. At each step $j$, the upper left $(j-1)$ block of $L_{k}$ is used to compute the first $j-1$ entries of the $j$ th column of $U_{k}$. In particular, computation of $N-M$ entries of the rightmost column uses only the upper left $(N-M)$-block of $L_{k}$, i.e. the block representing the $n c s$. Writing this last step of the algorithm explicitly, the $N-M$ first entries of column $N-M+1$ of $U_{k}$ satisfies

$$
L_{n c} \hat{U}_{k, \cdot(N-M+1)}=\hat{Y}_{\text {link }, \cdot k}
$$

where $\hat{Y}_{\text {link }, \cdot k}$ denotes the first $N-M$ entries of the column $Y_{\text {link }, \cdot, k}$. The vector $\hat{U}_{k, \cdot(N-M+1)}$ is therefore computed with a triangular backwards solve using the factorization of $Y_{n c}$ only. Similarly, we obtain the first $N-M$ entries of row $N-M+1$ of $L_{k}$ by the equation

$$
U_{n c}^{T} \hat{L}_{k,(N-M+1)}^{T}=\hat{Y}_{\text {link }, k}^{T} .
$$

again using only the factorization of $Y_{n c}$. Thus, using (6), we get $U_{k,(N-M+1, N-M+1)}$ from two backwards solutions using the factorization of $Y_{n c} .^{5}$

With the above computation, all matrices and operations involved are sparse and we completely avoid the fill-in producing factorization of the full admittance matrix $Y$. In addition, only the backwards solutions are dependent on $k$, and the factorization of $Y_{n c}$ must be done only once. Due to the sparsity, the backwards solutions are each computationally

\footnotetext{
${ }^{5}$ It is possible to reuse parts of the computations from the factorization step when solving (7) and (8). We will explore this further in future work.
}

lightweight, and they can in addition be computed completely in parallel. In the sequel, we denote the factorization of $Y_{n c}$ for the factorization step and the backwards solutions (7),(8) for the backwards-solve step. The algorithm for computing Thevenin impedances with this approach is listed in Algorithm 1. Though we will see in the experiments section that

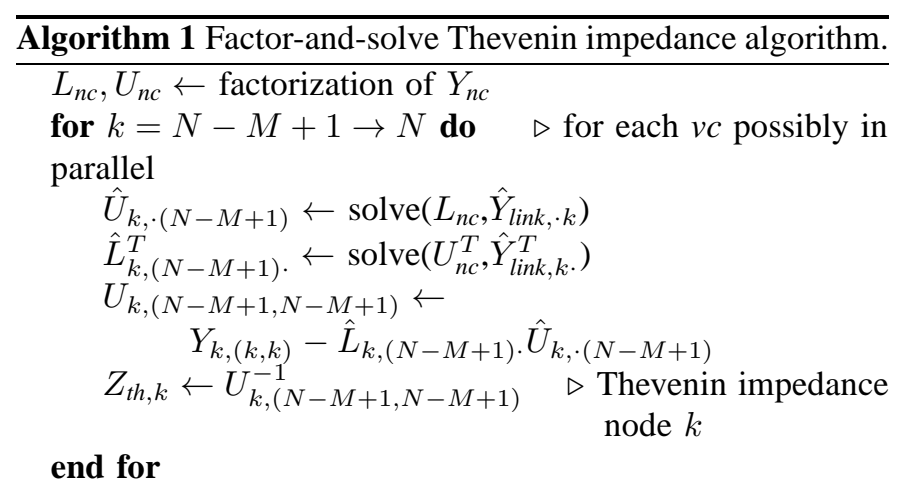

the backwards-solve step can dominate the runtime, the completely parallel nature of the loop over all $v c$ s makes speeding up this step straight-forward by splitting the computation of several compute cores. In contrast, the factorization step is hard to parallelize and therefore in reality the limiting factor of the algorithm. We analyse this step below.

\section{A. Node Elimination and Factorization Speed}

The factorization step of Algorithm 1 consist of the LUfactorization of $Y_{n c}$. We use the KLU solver [14] for the factorization in contrast to e.g. [5] which uses UMFPACK [24] when factoring $Y$. The sparsity of the network matrices are so high that KLU being a left-looking solver performs better than a right-looking multifrontal methods such as UMFPACK.

KLU is a state-of-the-art and very optimized solver, and it is therefore inherently difficult to improve the factorization speed. Nevertheless, it turns out we can speed up the factorization step of Algorithm 1 by using that we only need to solve (6) for which factorization of the full submatrix $Y_{n c}$ is not required. Instead, we perform node elimination prior to factorization to reduce the matrix size. We denote this part of the factor-and-solve algorithm the node elimination step.

Successive node elimination using the update formula (5) produces an equivalent network matrix that has fewer nodes but potentially is less sparse. For simulation of large resistor networks, several methods elimination parts of the system is used to reduce the size of the network as much as possible without producing to much fill-in [25], [26].

For real-time computation, we will see that node elimination can speed up the computation but only if careful consideration is taken with respect to the amount of fill-ins and the time used for elimination. Due to the efficiency of KLU, we can be quite relaxed in removing only a relatively limited number of nodes. We do this with a simple fill-in reducing strategy: we scan through the ncs removing a node only if it is connected to less than 4 other $n c s$ and if the fill introduced in the link matrix $Y_{\text {link }}$ is limited. Since removing nodes of degree 3 or less does 
not introduce fill-ins, this strategy ensures that the number of non-zeros in $Y_{n c}$ does not increase during the process, confer Figure 2. The number of non-zeros in $Y_{\text {link }}$ will in general increase but the number of added fill-ins is controlled by a fixed limit.

As we will see in the experiments section, the application of node elimination prior to running Algorithm 1 reduces the computational effort for the factorization step by a factor of $2-3$.

\section{EXPERIMENTS}

We will examine the speedup provided by the factor-andsolve Thevenin impedance algorithm and evaluate its absolute runtime. In particular, we will show that the Thevenin impedance of all generators for power systems of considerable sizes can be established in less than $3 \mathrm{~ms}$. and therefore be performed in real time. In addition, we will explore the runtime of the serial and parallel parts of the algorithm to evaluate the achieved overall efficiency, and we will show the great reduction in size and number of non-zeroes for the matrix to be factored.

We perform experiments on admittance matrices generated from test systems included in the PSS $\mathrm{R}$ - $30.0^{6}$ and MATPOWER [27] network simulation packages. The test systems include the US west-coast (1648 buses, 2602 branches) and US east-cost ( 7917 buses, 13014 branches) power grids along with 6 additional systems ranging from 2383 to 3120 buses $^{7}$.

The runtime is tested on a $3.2 \mathrm{GHz}$ Intel Core i7 hexa-core desktop CPU. In accordance with [5], UMFPACK [14] is used for factoring the full admittance matrix with the reference method, and KLU [14] is used for the factorization step of Algorithm 1. The main loop of the algorithm is parallelized using six threads, and the node elimination step uses AVX vector instructions to exploit fine-grained parallelism.

Figure 3 shows for each test system the runtime of the original Thevenin impedance algorithm employing LUfactorization of the full admittance matrix, the runtime of the factor-and-solve algorithm without node elimination, and the factor-and-solve algorithm with node elimination prior to the factorization. For all three approaches, the runtime of the initial symbolic pre-factorization step is left out of the measurements because this step only need to be done once for each network. The timings are performed just on the computational parts leaving out the time used for initial copying of data, and the obtained timings are averaged over a large number of runs. Please note the logarithmic scale on the vertical axis and the achieved approximately 80 times speedup on the largest system with the factor-and-solve algorithm compared to the previous method.

In Figure 4, we plot the runtime of the three different parts of the factor-and-solve algorithm: node elimination,

\footnotetext{
${ }^{6} \mathrm{http} / / / \mathrm{www} . \mathrm{energy}$ siemens.com/us/en/services/ power-transmission-distribution/power-technologies-international/ software-solutions/pss-e.htm

${ }^{7}$ The packages includes test systems that are considerably smaller. The runtime for these systems are negligible with the developed algorithm and therefore not included in the evaluation.
}

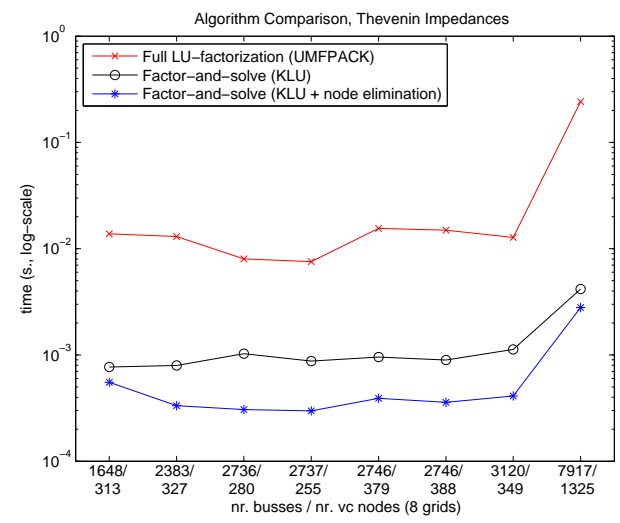

Figure 3. Computation time for determining Thevenin impedances using the full LU-factorization ([5], red), the factor-and-solve algorithm (black), and factor-and-solve algorithm with node elimination (blue). Evaluation performed on 8 power grids ranging from 1648 buses to 7917 buses with between 313 and 1325 voltage controlled nodes. Note the logarithmic scale on the time axis. For the largest system, the new method is roughly 80 times faster than the previous approach.

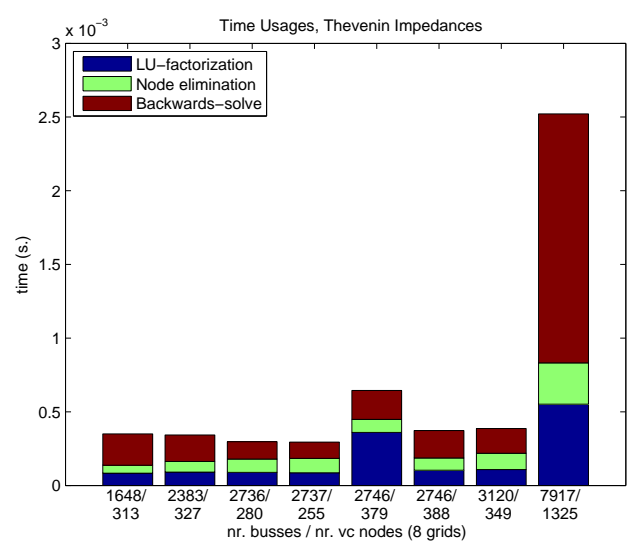

Figure 4. The time consumed for the three different parts of the factor-andsolve algorithm: factorization (blue), node elimination (green), and backwardssolve (red). The backwards-solve step parallelizes completely and the runtime can thus be reduced by employing more computational cores.

factorization, and backwards-solve. It is seen that a relatively large portion of the computational effort is spend on the backwards-solve. It is important to relate this to the fact that the backwards-solve step is completely parallelizable. For the results here, we used all 6 cores of the test machine. If a reduction in runtime is needed, a machine with more cores will allow the runtime of the backwards-solve step to be driven down below the runtime used for the reduction and factorization. In addition, there is room for more optimization of the code used for computing the backwards-solve.

Because backwards-solve step can be parallelized, the serial parts of the algorithm are in reality the true bottlenecks. In Figure 5, we plot the runtime of the serial parts in order to evaluate the benefits of the node elimination step. Employing node elimination results in a 2-3 times speedup for this part of the algorithm. In total, the factor-and-solve algorithm reduced the dimension of the matrix to be factorized for the largest 


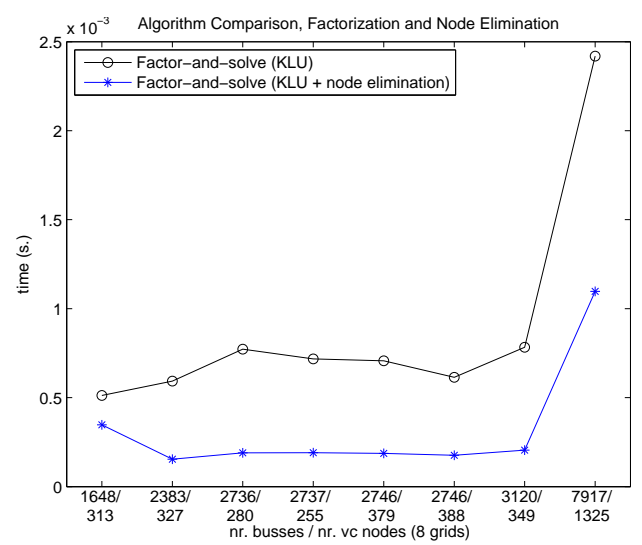

Figure 5. Runtime for the factor-and-solve algorithm excluding the backwards-solve step without node-elimination (black) and with nodeelimination (blue). Node elimination results in a speedup of a factor 2-3 for this part of the algorithm

test system from $7917 \times 7917$ (the full admittance matrix) to $960 \times 960$ (the node eliminated non-controlled part of the admittance matrix). At the same time, the number of non-zeros in the factors is reduced from 1549093 to 10174 .

\section{CONCLUSION}

Real-time calculation of Thevenin impedances is important for several suggested approaches to stability assessment. We have given theoretical arguments for why excessive fill-in occurs in the factorization used for previous approaches to calculating Thevenin impedances. These insights have lead to an improved algorithm that achieves an order of magnitude speedup and that allows parallelization of the computationally heavy part of the algorithm. Additional saving in computation time is in achieved by using node elimination prior to the factorization step.

On admittance matrices from several power systems, we evaluate and characterize the performance of the factor-andsolve algorithm on large and complex grids. Comparison with previous approaches shows approximately 80 times speedup for the largest power system. In addition, we determine how the different steps of the algorithm affect its performance and how the runtime can be controlled using parallelization of the backwards-solve step. As a result, for these systems, the Thevenin impedance computation is no longer a bottleneck for real-time transient stability assessment.

\section{REFERENCES}

[1] F. Li, W. Qiao, H. Sun, H. Wan, J. Wang, Y. Xia, Z. Xu, and P. Zhang, "Smart transmission grid: Vision and framework," IEEE Transactions on Smart Grid, vol. 1, no. 2, Sep. 2010.

[2] A. G. Phadke and J. S. Thorp, Synchronized Phasor Measurements and Their Applications, 1st ed. Springer, Sep. 2008.

[3] A. Phadke and R. de Moraes, "The wide world of wide-area measurement," IEEE Power and Energy Magazine, vol. 6, no. 5, Oct. 2008.

[4] M. Glavic and T. Van Cutsem, "Wide-area detection of voltage instability from synchronized phasor measurements. part i: Principle," Power Systems, IEEE Transactions on, vol. 24, no. 3, Aug. 2009.

[5] H. Jóhannsson, "Development of early warning methods for electric power systems," Ph.D. dissertation, Technical Univ. of Denmark, 2011.
[6] H. Johannsson, R. Garcia-Valle, J. Weckesser, A. Nielsen, and J. Ostergaard, "Real-time stability assessment based on synchrophasors," in PowerTech, 2011 IEEE Trondheim, Jun. 2011.

[7] H. Jóhannsson, J. Østergaard, and A. H. Nielsen, "Identification of critical transmission limits in injection impedance plane," International Journal of Electrical Power \& Energy Systems, vol. 43, no. 1, 2012.

[8] Y. V. Makarov, P. Du, S. Lu, T. B. Nguyen, J. Burns, and J. Gronquist, "Wide-area dynamic security region," in North American Power Symposium (NAPS), 2009, Oct. 2009.

[9] T. Weckesser, H. Jóhannsson, S. Sommer, and J. Østergaard, "Investigation of the adaptability of transient stability assessment methods to real-time operation," in IEEE PES ISGT Europe, Berlin, 2012.

[10] S. Corsi and G. Taranto, "A real-time voltage instability identification algorithm based on local phasor measurements," IEEE Transactions on Power Systems, vol. 23, no. 3, Aug. 2008.

[11] L. Warland and A. Holen, "Estimation of distance to voltage collapse: Testing an algorithm based on local measurements," in PSCC, Sevilla, 2002.

[12] I. Smon, G. Verbic, and F. Gubina, "Local voltage-stability index using tellegen's theorem," in IEEE Power Engineering Society General Meeting, 2007, Jun. 2007.

[13] K. Vu, M. Begovic, D. Novosel, and M. Saha, "Use of local measurements to estimate voltage-stability margin," IEEE Transactions on Power Systems, vol. 14, no. 3, Aug. 1999.

[14] T. A. Davis and E. Palamadai Natarajan, "Algorithm 907: KLU, a direct sparse solver for circuit simulation problems," ACM Trans. Math. Softw., vol. 37, no. 3, Sep. 2010.

[15] M. Ilic'-Spong, M. L. Crow, and M. A. Pai, "Transient stability simulation by waveform relaxation methods," Power Systems, IEEE Transactions on, vol. 2, no. 4, Nov. 1987.

[16] F. Dorfler and F. Bullo, "Kron reduction of graphs with applications to electrical networks," IEEE Transactions on Circuits and Systems, 2011.

[17] F. Pruvost, T. Cadeau, P. Laurent-Gengoux, F. Magoules, and F.-X. Bouchez, "Numerical accelerations for power systems transient stability simulations," in 17th Power System Computation Conference, 2011.

[18] T. H. Cormen, C. E. Leiserson, R. L. Rivest, and C. Stein, Introduction To Algorithms. MIT Press, 2001.

[19] T. A. Davis, Direct Methods for Sparse Linear Systems. SIAM, Sep. 2006.

[20] P. R. Amestoy, T. A. Davis, and I. S. Duff, "Algorithm 837: AMD, an approximate minimum degree ordering algorithm," ACM Trans. Math. Softw., vol. 30, no. 3, Sep. 2004.

[21] F. Zhang, Ed., The Schur Complement and Its Applications, ser. Numerical Methods and Algorithms, 2005, vol. 4.

[22] Y. Saad, Iterative Methods for Sparse Linear Systems. SIAM, Apr. 2003.

[23] Y. Saad and M. Sosonkina, "Distributed schur complement techniques for general sparse linear systems," SIAM J. SCI. COMPUT, vol. 21, 1997.

[24] T. A. Davis, "Algorithm 832: UMFPACK v4.3-an unsymmetric-pattern multifrontal method," ACM Trans. Math. Softw., vol. 30, no. 2, Jun. 2004.

[25] J. Rommes and W. H. A. Schilders, "Efficient methods for large resistor networks," IEEE Transactions on Computer-Aided Design of Integrated Circuits and Systems, vol. 29, no. 1, Jan. 2010.

[26] Z. Ye, D. Vasilyev, Z. Zhu, and J. Phillips, "Sparse implicit projection (SIP) for reduction of general many-terminal networks," in IEEE/ACM International Conference on Computer-Aided Design, Nov. 2008.

[27] R. Zimmerman, C. Murillo-Sanchez, and R. Thomas, "MATPOWER: steady-state operations, planning, and analysis tools for power systems research and education," IEEE Transactions on Power Systems, vol. 26, no. 1, Feb. 2011. 